\title{
Pyramiding PvPGIP2 and TAXI-III But Not PvPGIP2 and PMEI Enhances Resistance Against Fusarium graminearum
}

\section{Silvio Tundo, ${ }^{1}$ Raviraj Kalunke, ${ }^{1}$ Michela Janni, ${ }^{1}$ Chiara Volpi, ${ }^{1}$ Vincenzo Lionetti, ${ }^{2}$ Daniela Bellincampi, ${ }^{2}$ Francesco Favaron, ${ }^{3}$ and Renato D'Ovidio'}

\begin{abstract}
${ }^{1}$ Dipartimento di Scienze Agrarie e Forestali (DAFNE) Università della Tuscia, Via S. Camillo de Lellis snc, 01100 Viterbo; 2Dipartimento di Biologia e Biotecnologie "Charles Darwin", Sapienza Università di Roma, Piazzale Aldo Moro, 5, 00185 Roma, Italy; and ${ }^{3}$ Dipartimento Territorio e Sistemi Agro-Forestali (TeSAF), Research group in Plant Pathology, Università di Padova, Viale dell'Università 16, 35020 Legnaro (PD), Italy
\end{abstract}

Accepted 24 June 2016.

\begin{abstract}
Plant protein inhibitors counteract the activity of cell walldegrading enzymes (CWDEs) secreted by pathogens to breach the plant cell-wall barrier. Transgenic plants expressing a single protein inhibitor restrict pathogen infections. However, since pathogens secrete a number of CWDEs at the onset of infection, we combined more inhibitors in a single wheat genotype to reinforce further the cell-wall barrier. We combined polygalacturonase (PG) inhibiting protein (PGIP) and pectin methyl esterase inhibitor (PMEI), both controlling the activity of PG, one of the first CWDEs secreted during infection. We also pyramided PGIP and TAXI-III, a xylanase inhibitor that controls the activity of xylanases, key factors for the degradation of xylan, a main component of cereal cell wall. We demonstrated that the pyramiding of PGIP and PMEI did not contribute to any further improvement of disease resistance. However, the presence of both pectinase inhibitors ensured a broader spectrum of disease resistance. Conversely, the PGIP and TAXI-III combination contributed to further improvement of Fusarium head blight (FHB) resistance, probably because these inhibitors target the activity of different types of CWDEs, i.e., PGs and xylanases. Worth mentioning, the reduction of FHB symptoms is accompanied by a reduction of deoxynivalenol accumulation with a foreseen great benefit to human and animal health.
\end{abstract}

The plant cell wall $(\mathrm{CW})$ represents the first line of defense against pathogens. Cereal CW (Type II CW) consists of a network

Silvio Tundo and Raviraj Kalunke were equal contributors.

Current address for Raviraj Kalunke: Plant Molecular Biology Unit, Division of Biochemical Sciences, CSIR-National Chemical Laboratory, Pune 411008, Maharashtra, India.

Current address for Michela Janni: Institute of Bioscience and BioresourcesCNR, Via Amendola 165/A, 70126 Bari, Italy.

Current address for Chiara Volpi: Enza Zaden Italia Research SRL, S.S. Aurelia km 96+710, 01016 Tarquinia (VT), Italy.

Corresponding author: R. D’Ovidio; E-mail: dovidio@unitus.it

*The $\boldsymbol{e}$-Xtra logo stands for "electronic extra" and indicates that two supplementary figures are published online.

(c) 2016 The American Phytopathological Society of cellulose fibers embedded in a matrix of hemicelluloses, such as arabinoxylan and mixed linkage glucans, with a minor amount of xyloglucan and pectins (Vogel 2008). Pectins (5 to $10 \%$ ) are complex polymers with different structural domains including homogalacturonan, rhamnogalacturonan I, rhamnogalacturonan II, and xylogalacturonan. To overcome this barrier and colonize host tissues, pathogens secrete a number of cell wall-degrading enzymes (CWDEs), some of which have been demonstrated to be virulence factors (Kubicek et al. 2014). To counteract the activity of CWDEs, plant CW contains protein inhibitors that block, in a specific manner, the activity of these microbial enzymes (Juge 2006). Among these inhibitors, PG-inhibiting protein (PGIP) controls the activity of endopolygalacturonase (endo-PG; EC 3.2.1.15) (Kalunke et al. 2015), which is one of the first CWDEs produced during the infection process and is a virulence factor for some fungi, such as Botrytis cinerea, Aspergillus flavus, Alternaria citri, Claviceps purpurea, and Sclerotinia sclerotiorum (Isshiki et al. 2001; Li et al. 2004; Oeser et al. 2002; Shieh et al. 1997; ten Have et al. 1998), and bacteria, such as Agrobacterium tumefaciens, Ralstonia solanacearum, and Xylella fastidiosa (Rodriguez Palenzuela et al. 1991; Roper et al. 2007; Tans-Kersten et al. 2001).

The effectiveness of PGIP to improve disease resistance has been reported in different crops, including wheat and rice, that possess a CW with a poor content of pectin (Kalunke et al. 2015). Transgenic bread wheat (Triticum aestivum) lines overexpressing the bean PvPGIP2 showed reduced disease symptoms when challenged with the fungi Bipolaris sorokiniana (Janni et al. 2008) and Fusarium graminearum (Ferrari et al. 2012). Similarly, transgenic bread wheat expressing the soybean GmPGIP3 showed and improved resistance to the fungal pathogens Gaeumannomyces graminis var. tritici and B. sorokiniana (Wang et al. 2015a), and the overexpression of OsPGIP1 in transgenic rice enhanced resistance against Rhizoctonia solani in field experiments (Wang et al. 2015b). The observed improved resistance of PvPGIP2 plants has been associated with the capacity of PGIP to inhibit the activity of PGs secreted by the pathogens. This correlation was further reinforced by the observation that wheat plants expressing the bean PvPGIP2 did not enhance resistance against the biotrophic fungal pathogen Claviceps purpurea, which secretes two endo-PGs (CpPG1 and CpPG2) that are not inhibited by PvPGIP2 (Volpi et al. 2013).

PG activity is also conditioned by the level of methyl esterification of the $\mathrm{CW}$ pectin. High methyl esterification makes pectin less susceptible to degradation by PG (Bonnin et al. 2002; Limberg et al. 
2000; Lionetti et al. 2010; Volpi et al. 2011; Willats et al. 2001). The methyl esterification of pectin is mainly controlled by pectin methyl esterases (PME; EC3.1.1.11) (Pelloux et al. 2007; Willats et al. 2001). PME activity, which catalyzes the demethyl esterification of the C6 linked methyl ester group of homogalacturonan, the main pectic component of the cell wall, is tightly regulated by the PME inhibitor (PMEI) (Giovane et al. 2004; Lionetti et al. 2012).

The effectiveness of PMEI in controlling the endogenous PME activity and increasing the level of pectin methyl esterification has been first demonstrated in planta by overexpressing AtPMEI-1 or AtPMEI-2 inhibitors in Arabidopsis. These transgenic plants showed a significant reduction of symptoms caused by Botrytis cinerea and Pectobacterium carotovorum (Lionetti et al. 2007). Subsequently, the efficacy of a kiwi PMEI (AcPMEI) in limiting fungal infection was demonstrated in durum wheat against $B$. sorokiniana, $F$. graminearum, and $C$. purpurea (Volpi et al. 2011, 2013). The improved resistance of these transgenic plants against bacterial and fungal pathogens was related to the impaired ability of these pathogens to grow on methylesterified pectin and to a reduced capacity of their PGs to hydrolyze methyl-esterified pectin. Recently, the PMEI has been also demonstrated to restrict Tobacco mosaic virus and Turnip vein clearing virus in tobacco and Arabidopsis, respectively. PMEI could limit the interaction of PME with viral movement proteins hindering the enlargement of plasmodesmata and cell-to-cell viral spreading (Lionetti et al. 2014).

Another class of glycosidase inhibitors that counteract CWDEs is the xylanase inhibitors (XIs) (Dornez et al. 2010). These proteins inhibit the activity of microbial xylanases that are key enzymes in the degradation of arabinoxylans, a main component of the cereal $\mathrm{CW}$, and are virulent factors for the fungal pathogens Botrytis cinerea (Brito et al. 2006) and Mycosphaerella graminicola (Siah et al. 2010). Recently, XIs have been proven to limit disease symptom development. In particular, the overexpression of TAXI-III, an XI-inhibiting xylanase of glycoside hydrolase family 11 , in durum wheat transgenic plants reduced disease symptoms caused by F. graminearum (Moscetti et al. 2013). TAXI-III could protect wheat from $F$. graminearum via either direct inhibition of xylanase activity, by preventing host cell death, or both (Moscetti et al. 2015; Tundo et al. 2015).

Enhanced resistance by ectopic expression of XIs has also been obtained in rice plants overexpressing RIXI or OsHI-XIP. The rice plants overexpressing RIXI showed improved resistance against the fungal pathogen Magnaporte oryzae (Hou et al. 2015), whereas those overexpressing OsHI-XIP showed reduced larval infestation of the rice striped stem borer (Chilo suppressalis) and a decreased feeding and oviposition preferences of the rice brown planthopper (Nilaparvata lugens) (Xin et al. 2014).

On the basis of these remarkable results using single inhibitors and considering that pathogens secrete a composite mixture of CWDEs, in the present study, we investigated the pyramiding of PvPGIP2, AcPMEI, and TAXI-III to reinforce further the cellwall barrier and promote a greater improvement of wheat resistance. We focused our attention on fungal pathogens with a special consideration of $F$. graminearum, the causal agent of the devastating worldwide disease Fusarium head blight (FHB). We demonstrated also the effectiveness of PvPGIP2 in improving durum wheat resistance and verified the effect on $\mathrm{CW}$ composition of the single and combined expression of the glycosidase inhibitors PvPGIP2, AcPMEI, and TAXI-III.

\section{RESULTS}

\section{Transgenic durum wheat plants expressing PvPGIP2} show reduced leaf blotch symptoms.

In order to combine PvPGIP2 and AcPMEI, we first produced transgenic durum wheat plants expressing PvPGIP2.
This preliminary step was necessary because only bread wheat transgenic lines expressing PvPGIP2 were available. In total, 1,656 immature embryos of Triticum durum cv. Svevo were cotransformed, using pUbi::Pvpgip2 and pUbi::bar. A total of $33 \mathrm{~T}_{0}$ independent transgenic lines resistant to bialaphos and containing the Pvpgip2 gene were obtained, for a cotransformation efficiency of $2.3 \%$. The regenerated plants carrying the transgene were characterized for the accumulation of PvPGIP2 and for the endo-PG inhibition activity. Total protein extracts from leaves of three selected transgenic lines, MJ31-2, MJ3111 , and MJ31-17, and control plants were subjected to Western blotting analysis using an antibody raised against the bean PGIP. A clear immunodecoration signal corresponding to PvPGIP2 was detected in the transgenic plants, whereas no signal was detected in total protein extracts from the untransformed control plants (Supplementary Fig. S1A). The intensity of the signal varied between $T_{0}$ plants and was particularly strong in MJ31-2 and MJ31-11. The same leaf protein extracts were used to analyze the inhibition activity against an endo-PG of F. phyllophilum (FpPG). This enzyme is inhibited by PvPGIP2 while it is unaffected by the endogenous wheat PGIP (Janni et al. 2008). The analysis showed that the protein extract of the different transgenic lines inhibited FpPG with different efficacy that was related to the amount of PvPGIP2, as determined by Western blotting. In contrast, both the crude protein extract from the untransformed control plants and the boiled protein extracts of the transgenic lines did not affect the activity of FpPG, excluding the possibility of a nonproteinaceous inhibition (data not shown).

Genomic DNA blots of MJ31-11, using Pvpgip2 as probe, showed the expected 1-kb fragment after cleavage with BamHI, containing the entire coding region of Pvpgip2. Differently, the digestion with SphI, which cuts once within the pUbi::Pvpgip2, produced the expected hybridizing fragment of $5.7 \mathrm{~kb}$ plus additional fragments, most likely representing different insertion sites or rearranged copies of pUbi::Pvpgip2, as well as the terminal fragment of the transgene array.

Inhibition assays using total protein extracts from glumes and ovaries or anthers of MJ31-11 against FpPG showed that all these tissues have similar inhibition activity as that detected in leaves. Similarly, inhibition assays using extracellular fluids from seedlings of MJ31-11 showed the expected inhibition activity against FpPG, indicating that PvPGIP2 is accumulated in the apoplast. Conversely, extracellular fluids of untransformed control plants did not show any inhibition activity against FpPG.

In order to verify the capacity of durum wheat transgenic plants expressing PvPGIP2 to limit leaf blotch symptoms caused by $B$. sorokiniana, the $\mathrm{T}_{2}$ progeny of the MJ31-11 line (PGIP plants) and untransformed control plants of T. durum cv. Svevo (Svevo plants) were challenged with the pathogen. Inoculations with a conidial suspension were performed on the first emerged leaf and disease symptoms were recorded and evaluated at $72 \mathrm{~h}$ postinfection (hpi). Statistical analysis of the data showed a significant reduction $(P \leq 0.01)$ of about $50 \%$ of leaf blotch symptoms in MJ31-11 plants compared with Svevo plants. This result demonstrated that the expression of Pvpgip2 in durum wheat reduces leaf blotch symptoms to a similar extent as previously reported in transgenic bread wheats expressing this gene (Janni et al. 2008).

\section{Pyramided PGIP $\times$ PMEI plants show inhibition activities and a degree of pectin methyl esterification similar to that of parental plants.}

PGIP plants and the line MJ15-69 expressing AcPMEI (PMEI plants) (Volpi et al. 2011) were crossed and the $F_{1}$ seeds S010 (PGIP $\times$ PMEI plants) were screened by polymerase chain reaction (PCR) to select those carrying both transgenes 
(data not shown). The copresence of the two transgenes was also confirmed by Southern blot on genomic DNA of $F_{2}$ plants showing both PvPGIP2 and AcPMEI inhibition. DNA digestion with BamHI, which excises the entire coding sequence from the plasmid, shows the expected copresence of hybridization bands of about 1.0 and $0.5 \mathrm{~kb}$ for Pvpgip2 and Acpmei, respectively (Supplementary Fig. S2). The different intensity of the Pvpgip2 or Acpmei hybridization bands in the $\mathrm{F}_{2}$ samples was probably related to the homozygous or heterozygous condition of the transgene.

Total leaf protein extract from the $\mathrm{F}_{1}$ plants carrying both transgenes was used to analyze the inhibition activity of PvPGIP2 and AcPMEI. The activity of PvPGIP2 was analyzed against FpPG, whereas AcPMEI inhibition was determined by comparing endogenous PME activities of transgenic plants relative to Svevo plants. $F_{1}$ PGIP $\times$ PMEI plants showed inhibition of FpPG at a similar level to PGIP plants and the almost absence of endogenous PME activity as in the PMEI plants, indicating that both PvPGIP2 and AcPMEI were active in the cross progeny (Fig. 1A).

We also determined the uronic acid content and the degree of pectin methyl esterification (DM) in $\mathrm{CW}$ extracted from $\mathrm{F}_{4}$ PGIP $\times$ PMEI plants, parental plants, and cv. Svevo. All these plants showed a similar content of uronic acids (Table 1). The PGIP $\times$ PMEI plants and PMEI plants showed a significant $57 \%$ increase of DM compared with untransformed control and PGIP plants (Fig. 1B). No phenotypic differences with untransformed control plants or transgene silencing events have been detected up to the $\mathrm{F}_{4}$ generation.
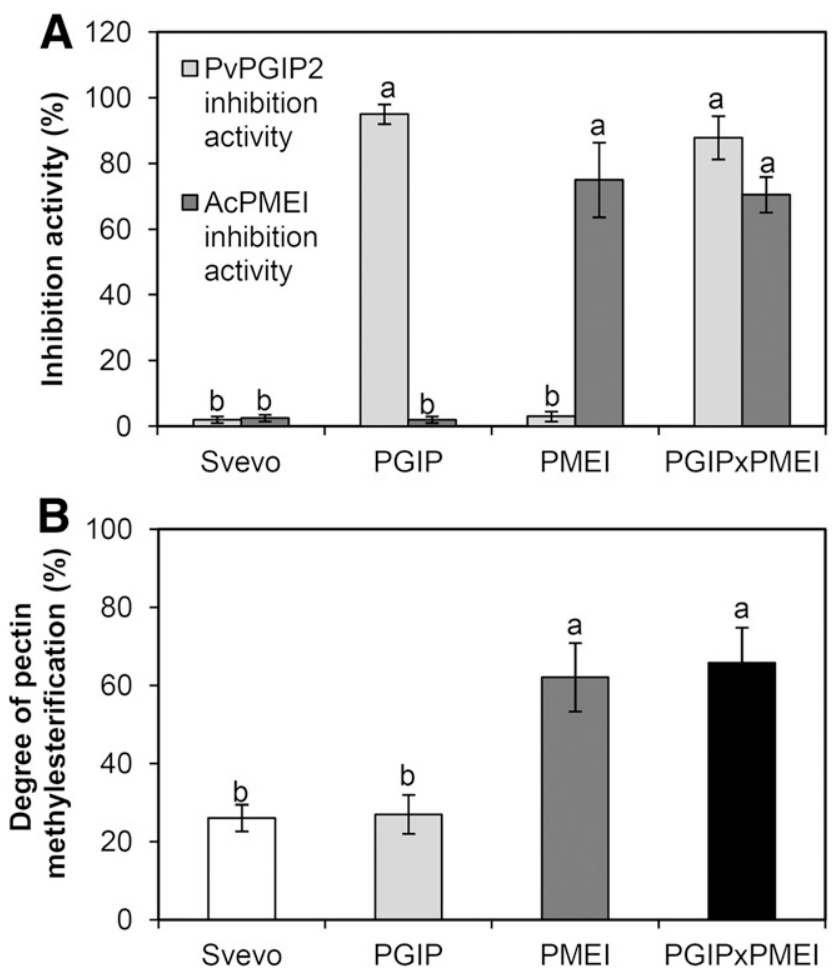

Fig. 1. Inhibition activity and degree of methyl esterification of 'Svevo' (control), cross PGIP $\times$ PMEI (polygalacturonase inhibiting protein and pectin methyl esterase inhibitor), and parental plants PGIP and PMEI. A, The bean PvPGIP2 inhibition activity was determined by using $10 \mu \mathrm{g}$ of total protein extract of leaf tissues against $0.0011 \mathrm{U}$ of Fusarium phyllophilum endo-polygalacturonase (FpPG). No inhibition activity against FpPG was detected in Svevo plants. Kiwi AcPMEI inhibition activity was tested against endogenous PME activity. Bars represent the average \pm standard error of at least 20 biological replicates. B, Degree of methyl esterification of cell-wall pectin isolated from leaf tissue. Letters above columns correspond to ranking of Tukey test $(P<0.05)$. Values represent the average \pm standard error of three independent experiments with three plants each.
Pyramided PGIP $\times$ PMEI plants show reduced leaf blotch, FHB, and ergot disease symptoms at levels similar to the parental plants.

The effect of the coexpression of PvPGIP2 and AcPMEI in PGIP $\times$ PMEI plants was analyzed against the fungal pathogens $B$. sorokiniana, $F$. graminearum, and $C$. purpurea. Infection with $B$. sorokiniana was analyzed on the first emerged leaf and disease symptoms were analyzed at $72 \mathrm{hpi}$. The $\mathrm{F}_{4} \mathrm{PGIP} \times \mathrm{PMEI}$ plants showed a significant reduction (about 50\%) in disease symptoms compared with Svevo plants (Fig. 2). However, pyramided plants showed no significant difference in disease symptoms compared with parental PGIP and PMEI plants, which showed a similar reduction of about $50 \%$ in disease symptoms compared with nontransgenic Svevo plants (Fig. 2).

Flowering spikes of PGIP $\times$ PMEI plants, PGIP plants, PMEI plants, and Svevo plants were infected with a conidial suspension of $F$. graminearum. FHB disease symptoms developed at $3 \mathrm{dpi}$ and the spread of the symptoms was visually scored for a period of 15 days. Four independent infection experiments on at least 12 plants per genotype were performed. We obtained two infection experiments showing a faster rate of FHB symptom development and two with a slower rate of disease symptom development (Fig. 3A and B), possibly due to a varying number of germinated conidia in the different conidial preparations. Therefore, the two groups of experiments were analyzed separately. In the experiments with faster development of symptoms (Fig. 3A), the reduction of FHB symptoms (25\%) in PGIP plants was significant from 7 to $9 \mathrm{dpi}$, as compared with Svevo plants.

Table 1. Quantification of uronic acids content of cell-wall pectin ${ }^{\mathrm{y}}$

\begin{tabular}{lc}
\hline Line & ${\text { Uronic } \text { acids }^{\mathbf{z}}}^{\text {Svevo }}$ \\
PGIP plants & $0.056 \pm 0.0004 \mathrm{a}$ \\
PMEI plants & $0.055 \pm 0.0008 \mathrm{a}$ \\
PGIP $\times$ PMEI plants & $0.057 \pm 0.0004 \mathrm{a}$ \\
\hline
\end{tabular}

y Isolated from 'Svevo' (control), cross PGIP $\times$ PMEI (polygalacturonase inhibiting protein and pectin methyl esterase inhibitor), and parent plants PGIP and PMEI.

${ }^{\mathrm{z}}$ Values shown as micromoles per milligram of cell wall. The same letter indicates no significant differences, according to one way analysis of variance and ranking of Tukey test at 0.95 confidence level and $P<0.05$ level of significance. Data represent average of three biological replicates.

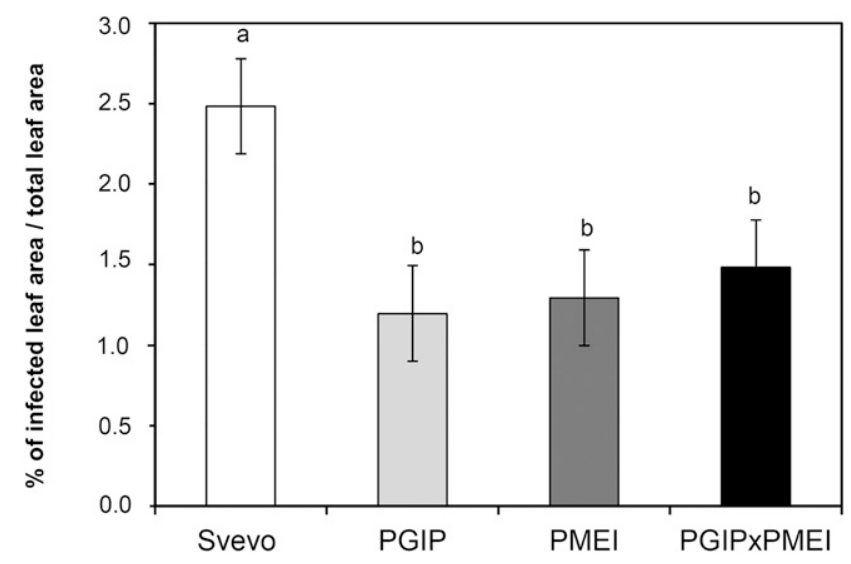

Fig. 2. Quantification of foliar spot blotch symptoms caused by Bipolaris sorokiniana on 'Svevo' (control), cross PGIP $\times$ PMEI (polygalacturonase inhibiting protein and pectin methyl esterase inhibitor), and parental plants PGIP and PMEI. Bars show averages and standard errors of the area of lesions divided by the total leaf area (square centimeters) of three independent experiments, each performed with at least 12 plants of each genotype. Different letters indicate data sets significantly different according to Tukey test $(P<0.05)$. 
Similarly, the PMEI plants showed a reduction of disease symptoms of about $30 \%$ as compared with Svevo plants. PGIP $\times$ PMEI plants showed a significant reduction of symptoms starting from 7 to $9 \mathrm{dpi}$, compared with Svevo plants. In particular, at 7 dpi, the reduction of FHB symptoms was about $35 \%$, whereas at later stages, no significant difference with cv. Svevo plants was observed (Fig. 3A). However, the PGIP $\times$ PMEI showed no significant difference in $\mathrm{FHB}$ symptoms compared with the parental lines (Fig. 3A).

In the experiments with a slower rate of development of symptoms (Fig. 3B), the reduction of FHB symptoms in the parental PGIP and PMEI plants was significant from 11 to $15 \mathrm{dpi}$, as compared with untransformed control plants, with a reduction of disease symptoms of about $28 \%$ and $30 \%$ at $15 \mathrm{dpi}$, respectively (Fig. 3B). Similarly, PGIP $\times$ PMEI plants showed a significant reduction of disease symptoms from 11 to $15 \mathrm{dpi}$, compared with Svevo plants. However, the extent of reduction (37\% at $15 \mathrm{dpi}$ ) was similar to that of parental lines (Fig. 3A and B).
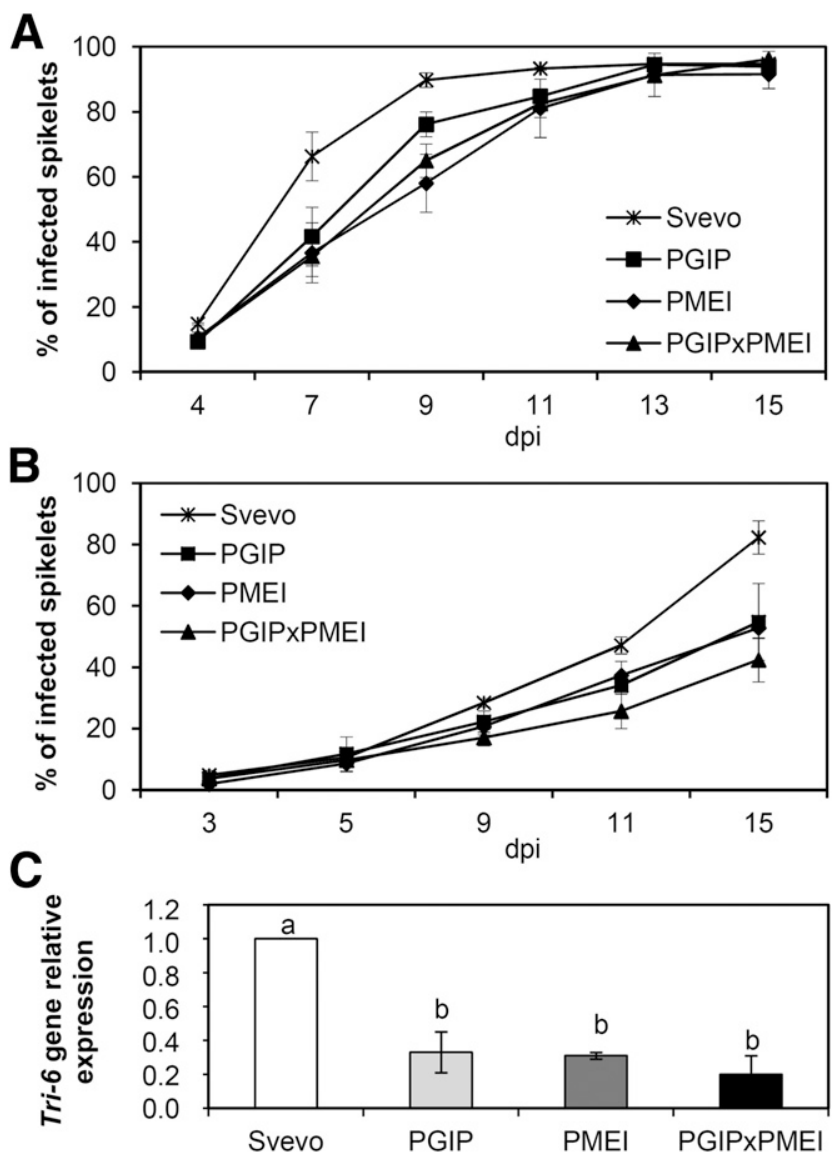

Fig. 3. Time-course analysis of Fusarium head blight (FHB) symptoms following Fusarium graminearum infection and quantification of fungal biomass in semolina of infected wheat spikes of 'Svevo' (control), cross PGIP $\times$ PMEI (polygalacturonase inhibiting protein and pectin methyl esterase inhibitor), and parental plants PGIP and PMEI. Experiments with A, high and $\mathbf{B}$, low rates of development of FHB symptoms are separately represented. Disease symptoms are expressed as percentage of spikelets showing symptoms on the total number of spikelets per spike. Data represent the average and standard errors of two experiments performed with at least 12 plants of each genotype. The average values are significantly different according to one way analysis of variance and ranking of Tukey test at $P<0.05$. dpi $=$ days postinfection. $\mathbf{C}$, Relative quantification of fungal DNA in wheat flour was calculated by quantitative polymerase chain reaction analysis of the Tri- 6 gene of $F$. graminearum. Actin was used as housekeeping gene. Bars represent average values \pm standard errors of two biological replicates and six technical replicates. Letters above columns correspond to ranking of Tukey test at $P<0.05$.
To verify fungal biomass accumulation, quantitative (q)PCR analysis was performed using specific primers for the fungal Tri-6 gene (Horevaj et al. 2011) on total DNA extracted from whole semolina of the parental and pyramided lines and Svevo plants. We analyzed both the infection experiments with faster and with slowed development of symptoms, and we found that the PGIP and PMEI samples derived from the experiment with faster development of symptoms contained a higher level of fungal biomass (0.38 and 0.29 relative expression to Svevo, respectively) compared with corresponding samples derived from the experiment showing a slower rate of fungal disease symptom development $(0.19$ and 0.12 relative expression to Svevo, respectively). Conversely, samples from the pyramided plants did not show any significant difference between the faster or slower rate of symptom development ( 0.33 and 0.29 relative expression to Svevo, respectively). The averaged values obtained from the two different infection experiments, i.e., with faster or slower rate of disease development, showed that samples from parental and pyramided plants exhibited about twofold reduced fungal biomass $(0.20 \pm 0.11,0.33 \pm 0.12$, and $0.31 \pm 0.02$ for PGIP, PMEI, and PGIP $\times$ PMEI plants, respectively) compared with Svevo plants (Fig. 3C). Thus, the reduced FHB symptoms were associated with a reduced accumulation of fungal biomass in the caryopses of parental and pyramided plants, possibly due to a reduced growth of the fungal pathogen in the early or advanced phases of infection (Fig. 3C).

A conidial suspension of the biotrophic fungal pathogen C. purpurea, the causal agent of ergot disease symptoms, was inoculated in flowering spikes of PGIP $\times$ PMEI, PGIP, PMEI, and Svevo plants. The infected plants showed honeydew (HD) production at about $5 \mathrm{dpi}$ and sclerotia were formed at about 20 dpi. Ergot disease symptoms were evaluated both by assessing the HD production on a scale from 1 to 4 and by determining the average number of sclerotia per spike in mature plants. The progression of fungal infection was evaluated as diseased spikelets out of the total number of inoculated spikelets per spike (Fig. 4A). All time points analyzed showed a significant reduction of diseased spikelets in PGIP $\times$ PMEI and PMEI plants as compared with Svevo and PGIP plants (Fig. 4A). For example, at $4 \mathrm{dpi}$, pyramided and PMEI plants showed only 5 to $6 \%$ of infected spikelets, whereas PGIP and Svevo plants showed 12 to $15 \%$ infected spikelets. The same extent of diseased spikelet reduction between these plants was maintained until the end of analysis at $14 \mathrm{dpi}$ (Fig. 4A). PGIP $\times$ PMEI and PMEI plants showed a reduced mean value of HD production at 7 and 9 dpi, as compared with PGIP and Svevo plants (Fig. 4B). For example, at $7 \mathrm{dpi}$ the average HD score was $2.6 \pm 0.7$ and $2.7 \pm 0.7$ for pyramided and PMEI plants, respectively, whereas that of PGIP and Svevo plants was $3.6 \pm 0.4$ and $4 \pm 0.0$, respectively (Fig. 4B). This reduced HD production and progression of fungal infection was also related to number of sclerotia per spike at 20 dpi. PGIP $\times$ PMEI $(4.7 \pm 1.9)$ and PMEI plants $(4.7 \pm 1.7)$ showed a significantly lower number of sclerotia per spike, as compared with PGIP $(8.4 \pm 1.7)$ and Svevo $(8.7 \pm 2.0)$ plants (Fig. 4C).

\section{Pyramided PGIP $\times$ TAXI plants show similar inhibition} activity as the parental transgenic plants.

Plants expressing PvPGIP2 and TAXI-III (PGIP $\times$ TAXI plants) were obtained from a cross (M011) between PGIP $\times$ PMEI plants and the transgenic line MJ30-23 expressing the Taxi-III gene (TAXI plants) (Moscetti et al. 2013). The copresence of Pvpgip2 and Taxi-III genes were confirmed by PCR, Southern blotting, and inhibition assay (Kalunke et al. 2013).

Inhibition activities of PGIP $\times$ TAXI plants, the parental PGIP and TAXI plants, and Svevo plants were tested against 
$0.0011 \mathrm{U}$ of FpPG and $0.005 \mathrm{U}$ of the xylanase AnM4Xyl, respectively. Pyramided plants showed high levels of inhibition $(72.1 \pm 5.0 \%)$ against FpPG but significantly lower levels compared with PGIP plants $(96.0 \pm 2.5 \%)$ (Fig. 5). The inhibition of PGIP $\times$ TAXI plants against AnM4Xyl was $69.7 \pm 8.5 \%$, similar to the level of inhibition (74.5 $\pm 7.6 \%)$ of TAXI plants (Fig. 5). Untransformed control plants did not show inhibition activity against both FpPG and AnM4Xyl.

\section{PGIP $\times$ TAXI plants show reduced FHB symptoms} compared with parental PGIP and TAXI plants.

To analyze the effect of the coexpression of PvPGIP2 and TAXI-III in the pyramided plants, three independent infection experiments with $F$. graminearum were performed on PGIP $\times$ TAXI plants, the parental PGIP and TAXI plants, and cv. Svevo. Plants showed FHB disease symptoms at 3 dpi, and the spread
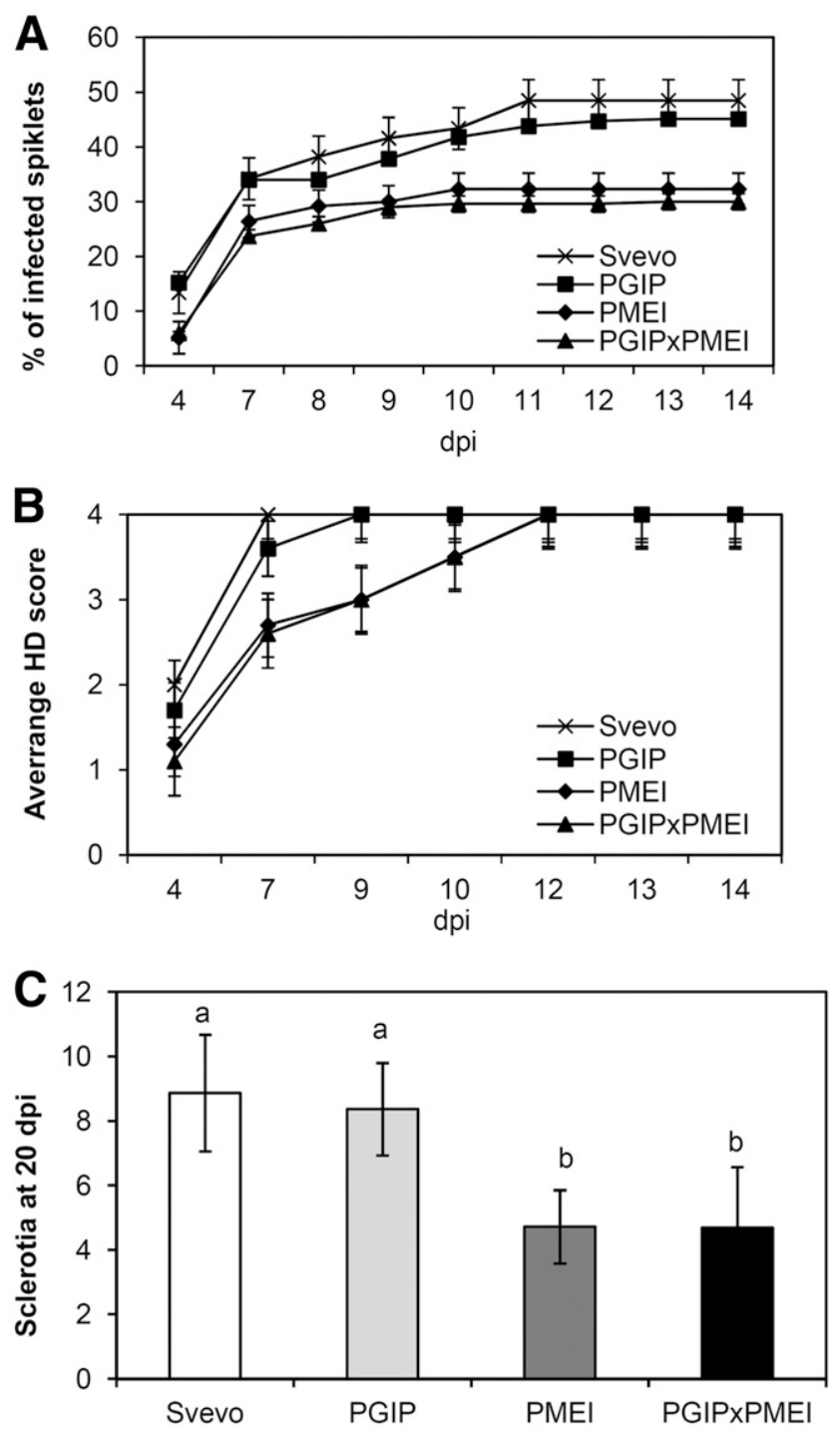

Fig. 4. Time-course analysis of ergot symptom development following the Claviceps purpurea infection of 'Svevo' (control), cross PGIP $\times$ PMEI (polygalacturonase inhibiting protein and pectin methyl esterase inhibitor), and parental plants PGIP and PMEI. A, Disease symptoms are expressed as percentage of infected spikelets out of the total number of inoculated spikelets per spike. B, Disease symptoms are expressed as honeydew (HD) production on a scale from 1 to 4 . C, Average number of sclerotia formation per spike at 20 days postinfection (dpi). At least 11 plants per genotype were used in the infection experiments. Letters above the columns correspond to ranking of Tukey test at $P<0.05$. of the symptom was visually scored for a period of 18 days (Fig. 6). Similarly to what observed in the infection experiments with the PGIP $\times$ PMEI plants, the three infection experiments on PGIP $\times$ TAXI plants showed two different rates of disease symptom development. One experiment showed a faster development of FHB symptoms compared with the other two experiments. In the infection experiments (Fig. 6A) with the faster rate of symptom development, the reduced FHB symptoms in the parental PGIP plants $(13 \%)$ was significant at 7 and 8 dpi (Fig. 6A), whereas PGIP $\times$ TAXI plants showed a significant reduction of symptoms between 7 and 14 dpi compared with Svevo plants. In particular, the reduction of FHB symptoms in the pyramided plants was maximized at $9 \mathrm{dpi}$, with a $22 \%$ reduction as compared with untransformed control plants (Fig. 6A). Moreover, PGIP $\times$ TAXI plants showed a significant reduction of FHB symptoms compared with PGIP plants from 8 to $14 \mathrm{dpi}$, with a maximum reduction of disease symptoms of $16 \%$ at 9 dpi (Fig. 6A).

In the other two infection experiments with a slower rate of symptom development (Fig. 6B), the reduction of FHB symptoms of PGIP plants (28\%) and TAXI plants (30\%), compared with Svevo plants, was significant from 13 to $18 \mathrm{dpi}$. The pyramided plants showed a significant reduction of FHB symptoms starting from 10 to $18 \mathrm{dpi}$, compared with Svevo plants. The reduction of symptoms was maximum at $18 \mathrm{dpi}$, with about $45 \%$ symptom reduction compared with Svevo plants (Fig. 6B). PGIP $\times$ TAXI plants also showed a significant difference in FHB symptoms compared with parental PGIP and TAXI plants from 10 to 18 dpi with a maximum reduction of 23 and $19 \%$ at $16 \mathrm{dpi}$, respectively (Fig. 6B).

To verify whether the difference in FHB symptoms was related to fungal biomass accumulation in infected caryopses, qPCR analysis was performed on DNA extracted from semolina of PGIP $\times$ TAXI, PGIP, TAXI, and Svevo plants. Results showed about a onefold reduction of fungal biomass in the parental PGIP and TAXI plants $(0.69 \pm 0.01,0.67 \pm 0.04$, respectively) compared with cv. Svevo, whereas the pyramided plants showed twofold reduction compared with cv. Svevo and onefold reduction compared with parent plants $(0.32 \pm 0.08)$ (Fig. 6C). Thus, the qPCR results confirmed that the reduced

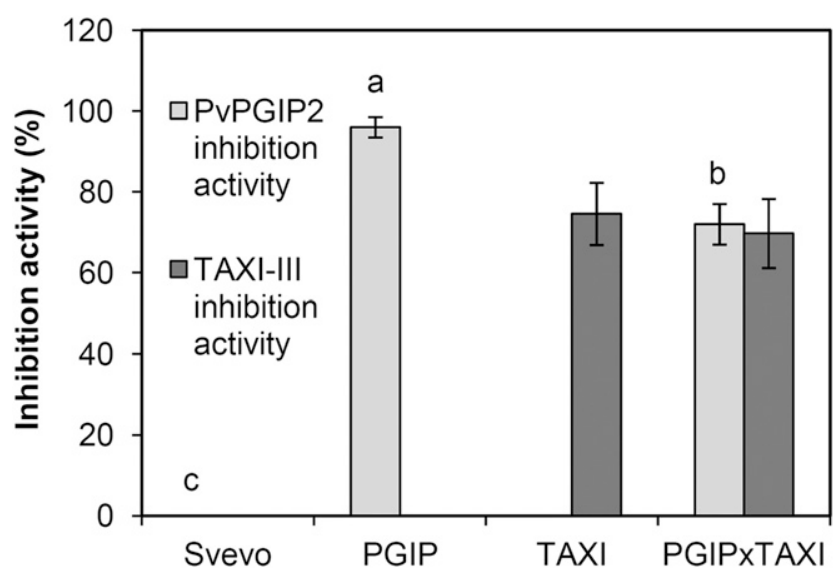

Fig. 5. Inhibition activities of 'Svevo' (control), cross polygalacturonase inhibiting protein and XI inhibiting xylanase (PGIP $\times$ TAXI), and parental plants PGIP and TAXI determined by agarose diffusion assay. PvPGIP2 inhibition activity was determined by using $10 \mu \mathrm{g}$ of total protein leaf extract against $0.0011 \mathrm{U}$ of FpPG. TAXI-III inhibition activity was determined by using $10 \mu \mathrm{g}$ of total protein leaf extract against $0.005 \mathrm{U}$ of AnM4Xyl. No inhibition activity was detected in untransformed control plants (cv. Svevo). Bars represent the average \pm standard error of at least 20 biological replicates. Letters above the columns correspond to ranking of Tukey test $(P<0.05)$. 
FHB symptoms corresponded to a reduced accumulation of fungal biomass. Moreover, the enhanced FHB resistance of PGIP $\times$ TAXI plants compared with parental PGIP and TAXI plants resulted in a further significant reduction of fungal biomass accumulation in the semolina of the pyramided plants (Fig. 6C).

In order to ascertain if the reduction of FHB symptoms and fungal biomass was related to a reduction of mycotoxin accumulations in the caryopsis, we quantified deoxynivalenol (DON) levels in the semolina of pyramided, parental, and control plants by liquid chromatography coupled to tandem mass spectrometry (LC-MS/MS). PGIP and TAXI plants showed a significant reduction of DON accumulation $(0.16 \pm 0.02 \mu \mathrm{g}$ and $0.07 \pm$ $0.00 \mu \mathrm{g} / \mathrm{mg}$ of semolina, respectively) compared with Svevo plants $0.24 \pm 0.02 \mu \mathrm{g} / \mathrm{mg}$ ). Moreover, DON accumulation in the semolina of pyramided plants $(0.03 \pm 0.00 \mu \mathrm{g} / \mathrm{mg})$ was significantly lower compared with the parental PGIP and TAXI plants.

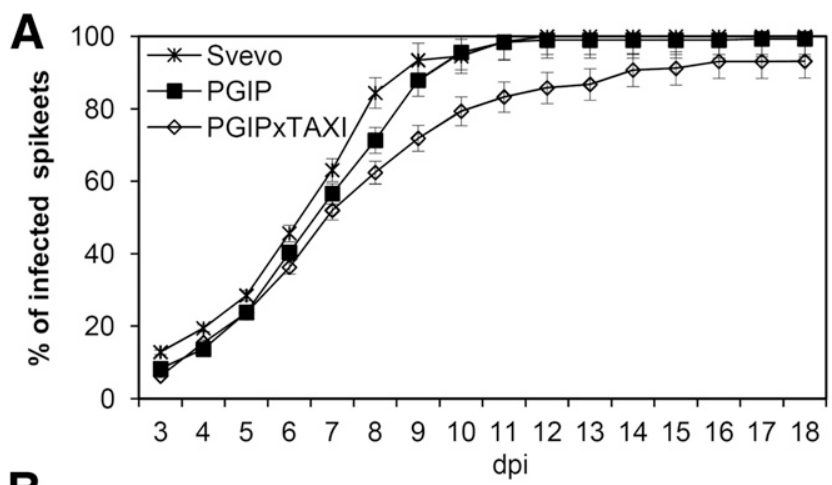

B
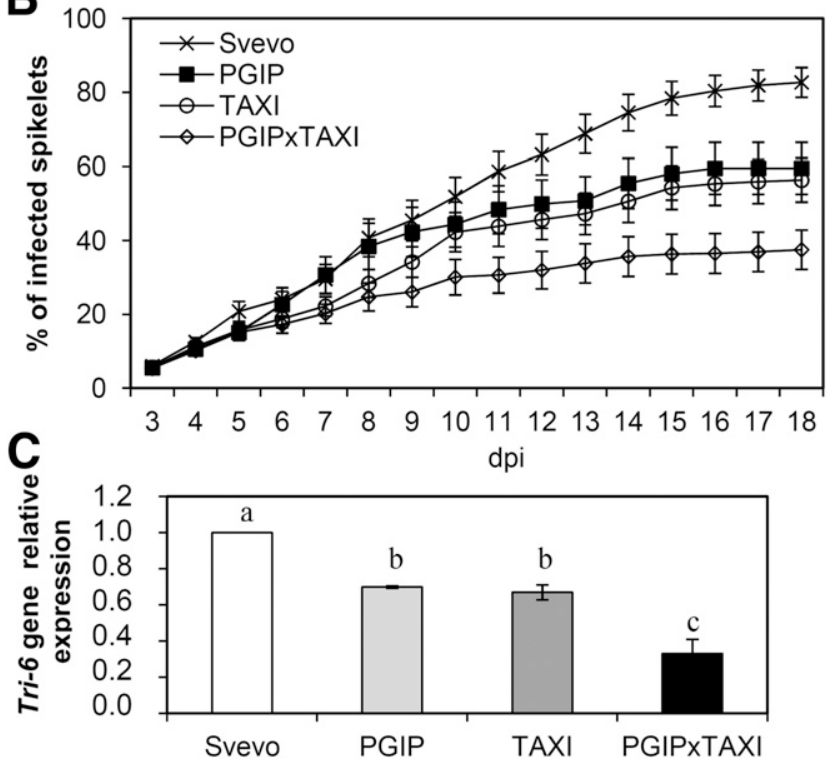

Fig. 6. Time-course analysis of Fusarium head blight (FHB) symptoms following Fusarium graminearum infection and quantification of fungal biomass in semolina of infected wheat spike of 'Svevo' (control), cross PGIP $\times$ TAXI (polygalacturonase inhibiting protein and xylanase inhibitor [XI] inhibiting xylanase), and parental plants PGIP and TAXI. Experiments with A, high and $\mathbf{B}$, low rates of development of FHB symptoms are separately represented. Disease symptoms are expressed as percentage of spikelets showing symptoms on the total number of spikelets per spike. Data represent the average and standard errors of one (A) and two (B) experiments performed with at least 12 plants of each genotype. The average values are significantly different according to one way analysis of variance and ranking of Tukey test at $P<0.05$. C, Relative quantification of fungal DNA in wheat flour was calculated by quantitative polymerase chain reaction analysis of the Tri-6 gene of $\mathrm{F}$. graminearum. Actin was used as housekeeping gene. Bars represent average values \pm standard errors of two biological replicates and six technical replicates. Letters above the columns correspond to ranking of Tukey test at $P<0.05$.
The overexpression of single glycosidase inhibitors or their combination causes changes in $\mathrm{CW}$ composition of wheat spikes.

To investigate if the overexpression of single or couples of $\mathrm{CW}$ enzyme inhibitors causes changes in CW composition and if these alterations could participate to the observed resistance phenotypes, the $\mathrm{CW}$ polysaccharides composition was determined on spikes of wheat PGIP, PMEI, TAXI, and PGIP $\times$ PMEI and PGIP $\times$ TAXI plants (Figs. 7 and 8). Monosaccharide composition of noncellulosic polysaccharides was determined by high performance anion exchange chromatography and pulsed amperometric detection (HPAEC-PAD) after acid hydrolysis of alcohol insoluble solid (AIS) (Figs. 7A and 8A). The analysis revealed xylose and arabinose as the main monosaccharides in this tissue, with lower amounts of glucose, galactose, galacturonic acid, rhamnose, glucuronic acid, and fucose. These results indicate that wheat spikes have a $\mathrm{CW}$ matrix enriched in hemicellulose polysaccharides, as observed in CW of other tissues of wheat (Gomez et al. 2008; Lionetti et al. 2015). The monosaccharide composition of PGIP and PMEI spikes shows significant differences in respect to Svevo plants (Figs. 7A and B). In particular, a significantly higher percentage of rhamnose, arabinose, galactose, glucose, galacturonic acid, and glucuronic acid, as well as a lower percentage of xylose was revealed in the resistant (parental lines and pyramided) plants as compared with the more susceptible cv. Svevo. A higher percentage of fucose was observed only in PMEI and in PGIP $\times$ PMEI plants. The PGIP $\times$ PMEI spikes present monosaccharide compositional changes comparable to those observed in the transgenic lines overexpressing the single inhibitors. Noteworthy, a further increase of the percentage of the pectic monosaccharides rhamnose, galactose, galacturonic acid, and glucuronic acid was detected in respect to plants overexpressing the single inhibitors. The arabinose to xylose ratio was significantly higher in the parental and pyramided plants with respect to Svevo plants (Fig. 7B). The amount of the crystalline cellulose was not significantly different between PGIP $\times$ PMEI, PGIP, PMEI, and Svevo plants (Fig. 7C). The monosaccharide composition of noncellulosic polysaccharides extracted from spikes of TAXI plants was similar to Svevo plants indicating that the overexpression of xylanase inhibitor does not alter the composition of matricial polysaccharides. In pyramided PGIP $\times$ TAXI plants, the same alterations observed in PGIP plants were detected (Figs. 8A and B). Interestingly, the amount of the crystalline cellulose was significantly higher in pyramided PGIP $\times$ TAXI plants in respect to Svevo plants and in respect to the plants expressing the single inhibitors (Fig. 8C). This result indicates that the combined overexpression of PvPGIP2 and TAXI-III affects the content of crystalline cellulose.

\section{DISCUSSION}

Plant pathogens exploit a number of CWDEs to overcome the cell-wall barrier, and each of them may contribute to a successful infection (Kubicek et al. 2014). Consequently, the reinforcement of the cell-wall barrier with diverse components facing CWDEs should guarantee a stronger and broader defense response. In the present study, we showed that the combination of PvPGIP2 and TAXI-III improved further durum wheat resistance against $F$. graminearum in comparison with the parental lines expressing, separately, each of these glycosidase inhibitors. We showed also that the pyramiding of PvPGIP2 and AcPMEI did not contribute to improve further durum wheat resistance against $F$. graminearum as compared with that already conferred by the presence of a single component.

Our first attempt to combine these glycosidase inhibitors was performed via genetic transformation by cobombardment of all three genes, Pvpgip2, Acpmei, and TAXI-III, plus the bar genes 
into durum wheat immature embryos. However, the high frequency of transgene silencing prevented the production of transgenic wheat lines expressing all three genes simultaneously (Kalunke et al. 2013). Probably the high copy number or the high constitutive expression of the transgenes-all of them were under control of the maize Ubi-1 promoter-was responsible for the observed silencing (Kalunke et al. 2013). The same result of multiple transgene silencing was obtained following our attempts to combine all three transgenes by classical crossing (Kalunke et al. 2013). Conversely the combination of two glycosidase inhibitors by traditional crossing was successful, with the combined inhibitors expressed at similar level as in the parental lines.

To perform these crossings, we first produced transgenic durum wheat lines expressing PvPGIP2, because this gene was available only in bread wheat lines (Ferrari et al. 2012; Janni et al. 2008). Similarly to what was obtained in the hexaploid bread wheat, we showed that the expression of PvPGIP2 is also effective in improving resistance of tetraploid durum wheat against $B$. sorokiniana (approximately $50 \%$ ) and $F$. graminearum (approximately 20 to $30 \%$ symptom reduction), indicating that the protective capacity of PvPGIP2 is not conditioned by the presence of the subgenome D peculiar of the hexaploid wheat.

The pyramided S010 plants containing AcPMEI and PvPGIP2 showed reduction of disease symptoms comparable to that of parental lines, indicating that PvPGIP2 or AcPMEI already, when expressed separately, contributed to the maximum attainable level of protection against $B$. sorokiniana and $F$. graminearum. This finding should be related to the capacity of PvPGIP2 and AcPMEI to protect the same cell-wall components, i.e., pectin. Moreover, the lack of any further increase of resistance in the pyramided PGIP $\times$ PMEI plants as compared with parental lines indicates that pectin degradation by fungal pathogens contributes only to a certain extent to the infection process. This result is in agreement with a previous observation obtained with AcPMEI plants and a $F$. graminearum PME-disrupted mutant (Sella et al. 2016). The mutant carrying the disrupted PME was less virulent on wheat spikes; however, its virulence was not reduced further on the AcPMEI plants (Sella et al. 2016).

Although the combined presence of PvPGIP2 and AcPMEI did not contribute to any further improvement of disease resistance, we showed that their pyramiding can be useful to broaden the spectrum of wheat protection against pathogens. This possibility rests on the observation that, although most PGs are less active on methylated pectins, there are some PGs, such as PGa and PGb of Aspergillus niger and Sclerotinia sclerotiorum, that are also active on methylated pectin (Favaron and Marciano 1992; Pařenicová et al. 2000). However, these PGs are inhibited by PvPGIP2 (D'Ovidio et al. 2006). Similarly, there are PGs that escape inhibition of PvPGIP2, such as PGs of $F$. verticilloides and $C$. purpurea, but their activity is strongly inhibited on methylated pectin (Raiola et al. 2008; Sella et al. 2004; Volpi et al. 2013). As proof of concept, we showed an improved resistance of the PGIP $\times$ PMEI plants against C. purpurea, which produced PGs not inhibited by PvPGIP2, as compared with the parental PvPGIP2 plants and cv. Svevo. The pyramided plants and the parental AcPMEI plants showed approximately $15 \%$ reduction of ergot symptoms and a significant reduced production of sclerotia compared with PvPGIP2 plants and cv. Svevo.

Differently to what was observed for the pyramiding of PvPGIP2 and AcPMEI, M011 hybrid plants containing the

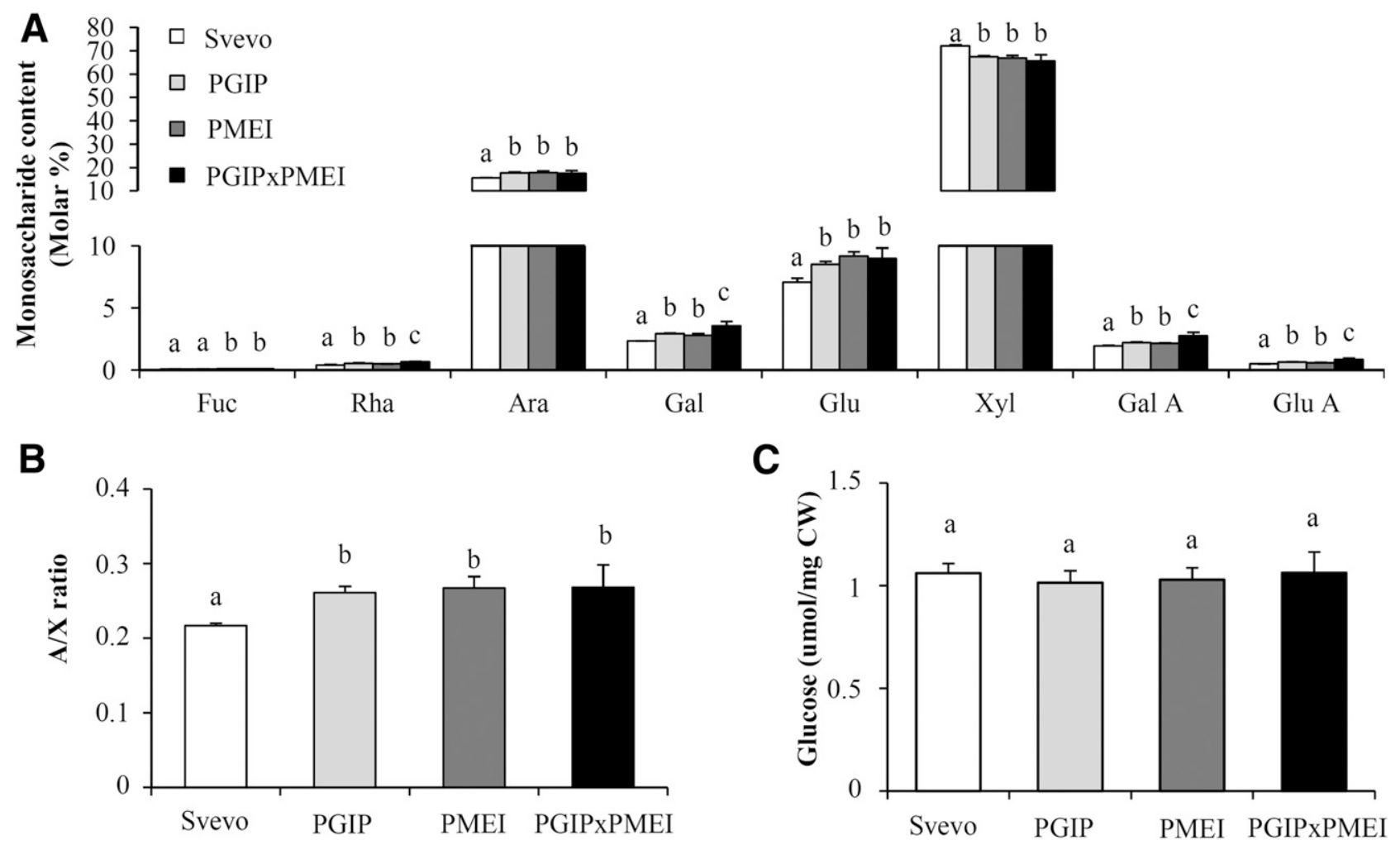

Fig. 7. Characterization of cell-wall polysaccharides in spikes of 'Svevo' (control), cross PGIP $\times$ PMEI (polygalacturonase inhibiting protein and pectin methyl esterase inhibitor), and parental plants PGIP and PMEI. A, Monosaccharide compositions. Fucose (Fuc), rhamnose (Rha), arabinose (Ara), galactose (Gal), glucose (Glc), xylose (Xyl), galacturonic acid (Gal A), and glucuronic acid (Glu A) released after 2 M triflouroacetic acid hydrolysis were determined by high performance anion exchange chromatography and pulsed amperometric detection. B, Quantification of Ara/Xyl ratio. C, Cellulose-derived glucose. Results represent the mean \pm standard deviation $(n=3)$. The different letters indicate data sets significantly different $(P<0.05)$ according to analysis of variance followed by Tukey test. 
combination PvPGIP2 and TAXI-III showed a higher level of resistance against $F$. graminearum (50\% symptom reduction) compared with the parental PGIP and TAXI plants.

This result at the phenotypic level was accompanied by a reduced growth of the pathogen, as indicated by the reduced amount of fungal biomass present in the semolina of the pyramided plants and by a lower amount of DON accumulation.

The enhanced resistance of plants expressing PvPGIP2 and TAXI-III can be explained by the efficacy of these inhibitors to prevent the degradation of different $\mathrm{CW}$ components, i.e., pectin and xylan, respectively, by the CWDEs secreted by the pathogen. However, the capacity of TAXI-III to prevent host cell death caused by xylanases secreted by $F$. graminearum (Moscetti et al. 2015) can also contribute to the enhanced resistance of the pyramided PGIP $\times$ TAXI plants.

An additional contribution to the improved resistance of the plants expressing both PvPGIP2 and TAXI-III may derive from the higher content of crystalline cellulose found in these plants. Interestingly, this increased amount of crystalline cellulose is peculiar to the PGIP $\times$ TAXI plants, suggesting a cooperative effect of the two glycosidase inhibitors in CW remodeling. We do not know how this can occur, however; both PGIP and TAXI bind to $\mathrm{CW}$ components, with the former binding specifically to pectin (Spadoni et al. 2006) and the latter to arabinoxylans (Fierens et al. 2008). Consequently, alteration in the amount of these inhibitors may influence the interaction between cell-wall components, especially when both of them are present.

Taken all together, these results indicate that the effectiveness of pyramiding of glycosidase inhibitors depends on their specific combination. Pyramiding of PvPGIP2 and AcPMEI does not enhance further wheat resistance against FHB, possibly because both inhibitors constrain the activity of the same CWDEs, i.e., PG secreted by the pathogen. Conversely, the pyramiding PvPGIP2 and TAXI-III supports a further improvement of resistance compared with plants carrying only PVPGIP2 or TAXI-III. Probably, the success of this combination rests on the capacity of PvPGIP2 and TAXI-III to constrain different targets, i.e., PGs and xylanases. Remarkably, the reduction of FHB symptoms is accompanied by a reduction of DON accumulation with a foreseen great benefit to human and animal health.

\section{MATERIAL AND METHODS}

\section{Plant material, genetic transformation, classical crosses, and DNA analyses.}

The MJ31-11 line expressing PvPGIP2 was obtained by cotransforming the immature embryos of Triticum durum cv. Svevo using plasmid pUBISR2 and pUbi::bar in a 1:3 molar ratio and following the procedures described by Janni et al. (2008). Plasmid pUBISR2 containing the coding region of Pvpgip2 (Leckie et al. 1999), including the N-terminal signal sequence, was inserted into the BamHI site of pAHC17 under control of the maize Ubiquitin1 promoter and Nopoline synthase (NOS) terminator. The pUbi::bar was used as selectable marker plasmid, which carries the bar gene that confers resistance to the bialaphos herbicide. The presence of the transgenes in the regenerated plants was verified by PCR analysis as reported by Janni et al. (2008).

Conventional crossing was performed between $\mathrm{T}_{2}$ plants of the line MJ31-11, containing Pvpgip2 transgene and $\mathrm{T}_{4}$ plants
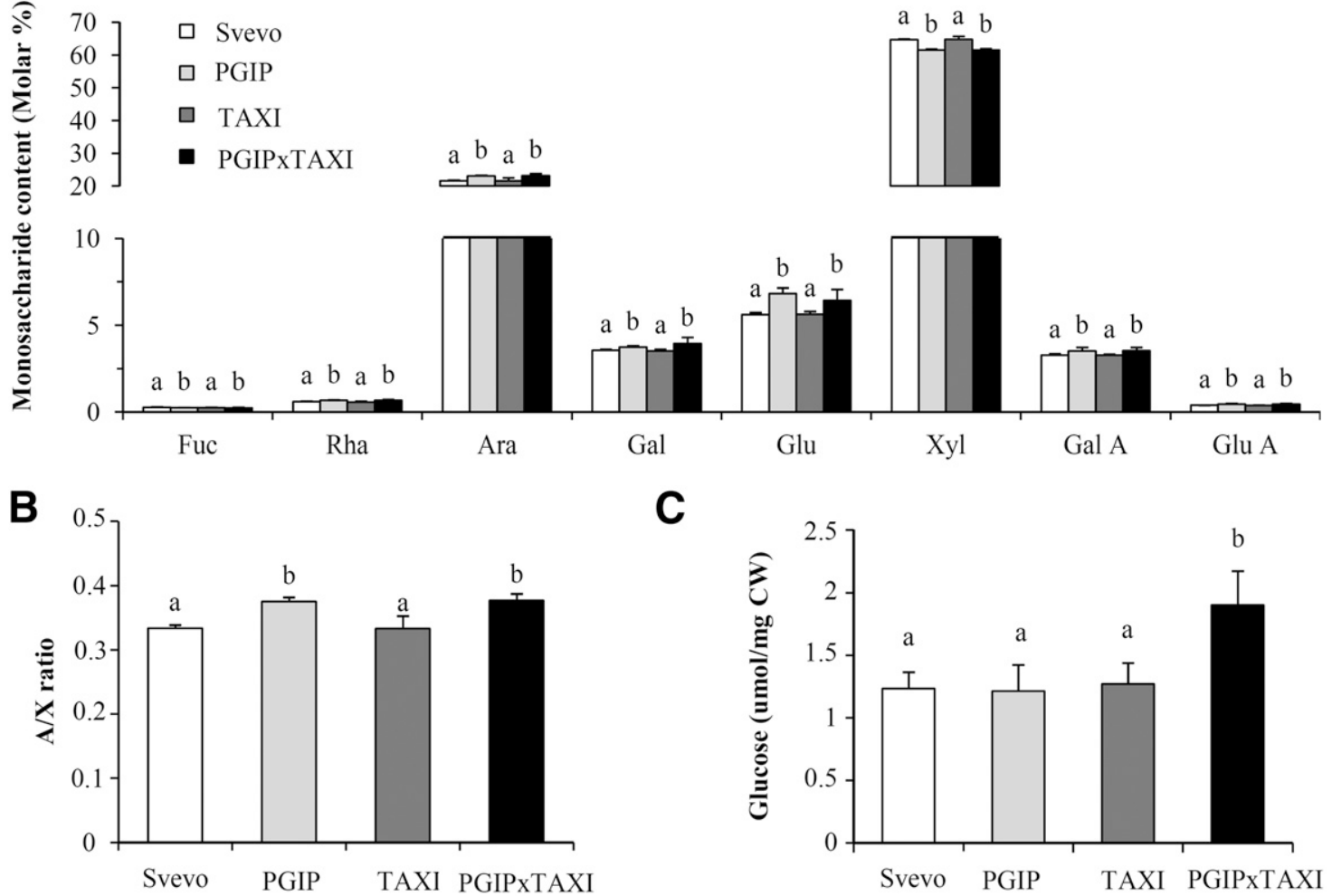

Fig. 8. Characterization of cell-wall polysaccharides in spikes of 'Svevo' (control), cross PGIP $\times$ TAXI (polygalacturonase inhibiting protein and xylanase inhibitor [XI] inhibiting xylanase), and parental plants PGIP and TAXI. A, Monosaccharide compositions. Fucose (Fuc), rhamnose (Rha), arabinose (Ara), galactose (Gal), glucose (Glc), xylose (Xyl), galacturonic acid (Gal A), and glucuronic acid (Glu A) released after 2 M triflouroacetic acid hydrolysis were determined by high performance anion exchange chromatography and pulsed amperometric detection. B, Quantification of Ara/Xyl ratio. C, Cellulose-derived glucose. Results represent the mean \pm standard deviation $(n=3)$. The different letters indicate data sets significantly different $(P<0.05)$ according to analysis of variance followed by Tukey test. 
of the line MJ15-69, containing Acpmei transgene (Volpi et al. 2011), to obtain S010 $F_{1}$ plants, which express simultaneously both PvPGIP2 and AcPMEI. PCR conditions to verify the presence of Pvpgip2 and Acpmei in the transgenic plants were those previously reported (Janni et al. 2008; Volpi et al. 2011).

Plants containing both transgenes were crossed again with transgenic line MJ30-23 expressing the Taxi-III gene (Moscetti et al. 2013) to obtain $\mathrm{F}_{1}$ M011 plants containing Pvpgip2, Acpmei, and Taxi-III as reported by Kalunke et al. (2013). Some $\mathrm{F}_{1} \mathrm{M} 011$ plants showed the copresence of only Pvpgip 2 and Taxi-III genes. These plants were used in this study. M011 plants containing all three transgenes showed only PvPGIP2 and TAXI-III inhibition activities, since Acpmei underwent silencing events (Kalunke et al. 2013).

Southern hybridization was carried out on green leaf material. Genomic DNA was extracted from 0.1 or $5.0 \mathrm{~g}$ of green leaf material following Tai and Tanksley (1990) or D'Ovidio et al. (1992). Genomic DNA (5 to $10 \mu \mathrm{g}$ ) was restrict-digested with BamHI or SphI enzyme and were separated on $1.2 \%$ agarose gel and transferred to positively charged nylon membranes (Roche Diagnostics), as described by Sambrook et al. (1989), and were hybridized with the coding region for each transgene labeled with digoxigenin (digoxigenin-11-uridine-50-triphosphate) (Roche Diagnostics), following the procedure of D'Ovidio and Anderson (1994).

Quantification of fungal biomass in infected caryopses was performed by qPCR by analyzing the Tri6 gene as reported by Moscetti et al. (2013). Two biological and three technical replicates were performed.

\section{Fungal PG, protein, and DON analyses.}

Fungal growth and endo-PG preparations were performed as previously described (Caprari et al. 1996) for F. phyllophilum. The crude protein extracts were obtained from leaves as previously described by D'Ovidio et al. (2004). Extracellular fluid was extracted from transgenic hypocotyls (Zadoks stage 47 ) by vacuum-infiltration with acetate buffer, as previously described (Salvi et al. 1990). The extracellular fluid had negligible glucose6-phosphate dehydrogenase activity, indicating that it was free of cytoplasmic contamination. Protein concentrations were determined with the Coomassie Plus-the Better Bradford assay kit (Pierce). Sodium dodecyl sulfate polyacrylamide gel electrophoresis and immunoblotting were performed as previously described (Desiderio et al. 1997). Polyclonal antibodies raised in rabbit against PGIP purified from Phaseolus vulgaris pods were used for immunoblotting experiments. Purified PvPGIP2 was obtained as previously reported (D'Ovidio et al. 2004).

The expression of PvPGIP2 at protein level in the transgenic wheat lines was detected by inhibition activity against FpPG, using an agarose diffusion assay as described by Janni et al. (2008). The expression of AcPMEI at the protein level in transgenic wheat lines was detected by inhibition activity against endogenous PMEs activity (Volpi et al. 2011). Xylanase inhibition activity of total protein extract was measured by agarose diffusion assay as reported by Moscetti et al. (2013). A total of $0.005 \mathrm{U}$ of Aspergillus niger endo- $\beta$-1,4-xylanase M4(AnM4xyl) (Megazyme) was used to check for TAXI-III inhibition activity. Metabolites were extracted from $100 \mathrm{mg}$ of wheat semolina in $\mathrm{C}_{2} \mathrm{H}_{3} \mathrm{~N}-\mathrm{H}_{2} \mathrm{O} 86: 14$ on a horizontal shaker (Finepcr) for $24 \mathrm{~h}$ at $180 \mathrm{rpm}$. The extracts were centrifuged at $10,000 \times g$ for $10 \mathrm{~min}$ and DON levels were ascertained by LC-MS/MS. The level of DON was determined in two $F$. graminearum infection experiments (i.e., those with faster disease development), using three technical replicates.

\section{Plant growth and infection assays.}

Seeds were surface sterilized with sodium hypochlorite $(0.5 \% \mathrm{vol} / \mathrm{vol})$ for $20 \mathrm{~min}$ and were then rinsed thoroughly in sterile water. Plants were vernalized at $4^{\circ} \mathrm{C}$ for 2 weeks and, then, were grown in single pots at 18 to $23^{\circ} \mathrm{C}$ with a 14-h light period $\left(300 \mu \mathrm{E} \mathrm{m}^{-2} \mathrm{~s}^{-1}\right)$.

The upper surface of each leaf of transgenic and control wheat plants in the first-leaf-emerged stage (Zadoks stage 11) were inoculated with $B$. sorokiniana 62608 DSMZ conidia. Procedure for $B$. sorokiniana fungal culture growth, conidia collection, infection with transgenic plants, and disease symptoms analysis at 72 hpi was carried out as described by Janni et al. (2008). Disease symptoms were evaluated as the ratio between leaf area showing symptoms and the total leaf area, expressed as a percentage.

To evaluate FHB disease symptoms, the opposite central spikelets in primary spikes (Zadoks stage 68) were inoculated with $F$. graminearum 3824 conidia. Procedure for the $F$. graminearum fungal cultures growth, conidia collection, infection with plants, and disease symptoms analysis was carried out as described by Volpi et al. (2011).

Three separate infection experiments with $B$. sorokiniana were performed on PGIP plants and five on PGIP $\times$ PMEI plants. For $F$. graminearum infection, four separate infection experiments were performed. In each separate infection, at least 12 plants for each genotype were used.

For $C$. purpurea infection experiments twelve plants per genotype were used in the infection experiment. Individual spikelets of each plant at Zadoks stage 59 were inoculated with a conidia suspension from a single isolate of $C$. purpurea. The conidia suspension of $C$. purpurea was prepared from concentrated HD of infected wheat spikes and was estimated using a Thoma hemocytometer and was adjusted to a final concentration of $1 \times 10^{5}$ conidia $/ \mathrm{ml}$. Procedure for the $C$. purpurea fungal cultures growth, conidia collection, infection with plants, and disease symptom evaluation was carried out as described by Volpi et al. (2013). A single infection experiment was performed with transgenic and control plants, using at least 11 plants for each genotype and by inoculating at least four spikelets per spike.

Infection data were subjected to one-way analysis of variance. When significant $F$ values were observed, a pairwise analysis was carried out by the Tukey honestly significant difference test (Tukey test) at a 0.95 confidence level.

\section{Isolation and analysis of $\mathbf{C W}$.}

Wheat spikes were collected at anthesis stage from wild type and transgenic lines, and AIS were isolated as previously described (Lionetti et al. 2015). Starch was removed by treating the AIS with the porcine Type I-A $\alpha$-amylase (100 U per gram of AIS) (Sigma-Aldrich) in a $100 \mathrm{mM}$ potassium phosphate buffer, $\mathrm{pH} 7.5,5 \mathrm{mM} \mathrm{NaCl}$, and $0.02 \%$ (wt/vol) $\mathrm{NaN} 3$ for $24 \mathrm{~h}$ at $37^{\circ} \mathrm{C}$. The suspension was centrifuged at $25,000 \times g$ for $20 \mathrm{~min}$, and the pellet was then washed with distilled water and $80 \%$ acetone. The monosaccharide composition of destarched AIS, hydrolyzed with $2 \mathrm{M}$ triflouroacetic acid, was determined by HPAEC-PAD, using a PA20 column (Dionex). Peaks were identified and quantified by comparison with a standard mixture of rhamnose, arabinose, fucose, galactose, glucose, xylose, galacturonic acid, and glucuronic acid (Sigma-Aldrich). The crystalline cellulose was determined as previously described (Updegraff, 1969). The cellulose-derived glucose content in destarched AIS was determined by an anthrone colorimetric assay (Scott and Melvin 1953) with glucose (Sigma G8270) as a standard. The degree of pectin methyl esterification and uronic acid content were determined as previously described (Lionetti et al. 2007).

\section{ACKNOWLEDGMENTS}

The authors thank A. Bitti for help in crossing the wheat plants. This research was supported by the Italian Ministry of University and Research 
(grant "Prin 2010-2011) and Italian Ministry of Agricultural, Food and Forestry Policies (grant "Progetto Internazionale di Sequenziamento del Genoma di Frumenti, Mappa fisica del Cromosoma 5A). R. M. Kalunke was supported by the Consorzio Interuniversitario per Biotecnologie (CIB), Trieste, Italy. M. Janni and S. Tundo were supported by 'Regione Lazio' (Grant 'Ricercatori a Tempo Determinato').

\section{LITERATURE CITED}

Bonnin, E., Le Goff, A., Körner, R., Vigouroux, J., Roepstorff, P., and Thibault, J. F. 2002. Hydrolysis of pectins with different degrees and patterns of methylation by the endopolygalacturonase of Fusarium moniliforme. Biochim. Biophys. Acta 1596:83-94.

Brito, N., Espino, J. J., and González, C. 2006. The endo- $\beta-1,4-x y l a n a s e$ xyn $11 \mathrm{~A}$ is required for virulence in Botrytis cinerea. Mol. Plant-Microbe Interact 19:25-32.

Caprari, C., Mattei, B., Basile, M. L., Salvi, G., Crescenzi, V., De Lorenzo, G., and Cervone, F. 1996. Mutagenesis of endopolygalacturonase from Fusarium moniliforme: Histidine residue 234 is critical for enzymatic and macerating activities and not for binding to polygalacturonaseinhibiting protein (PGIP). Mol. Plant-Microbe Interact 9:617-624.

D'Ovidio, R., and Anderson, O. D. 1994. PCR analysis to distinguish between alleles of a member of a multigene family correlated with wheat bread-making quality. Theor. Appl. Genet. 88:759-763.

D’Ovidio, R., Raiola, A., Capodicasa, C., Devoto, A., Pontiggia, D., Roberti, S., Galletti, R., Conti, E., O’Sullivan, D., and De Lorenzo, G. 2004. Characterization of the complex locus of bean encoding polygalacturonase-inhibiting proteins reveals subfunctionalization for defense against fungi and insects. Plant Physiol. 135:2424-2435.

D’Ovidio, R., Roberti, S., Di Giovanni, M., Capodicasa, C., Melaragni, M., Sella, L., Tosi, P., and Favaron, F. 2006. The characterization of the soybean polygalacturonase-inhibiting proteins (Pgip) gene family reveals that a single member is responsible for the activity detected in soybean tissues. Planta 224:633-645.

D’Ovidio, R., Tanzarella, O. A., and Porceddu, E. 1992. Isolation of an alpha-type gliadin gene from Triticum durum Desf. and genetic polymorphism at the Gli-2 locus. J. Genet. Breed. 46:41-48.

Desiderio, A., Aracri, B., Leckie, F., Mattei, B., Salvi, G., Tigelaar, H., Van Roekel, J. S. C., Baulcombe, D. C., Melchers, L. S., De Lorenzo, G., and Cervone, F. 1997. Polygalacturonase-inhibiting proteins (PGIPs) with different specificities are expressed in Phaseolus vulgaris. Mol. PlantMicrobe Interact. 10:852-860.

Dornez, E., Croes, E., Gebruers, K., De Coninck, B., Cammue, B. P. A., Delcour, J. A., and Courtin, C. M. 2010. Accumulated evidence substantiates a role for three classes of wheat xylanase inhibitors in plant defense. Crit. Rev. Plant Sci. 29:244-264.

Favaron, F., and Marciano, P. 1992. Polygalacturonase regulation in Sclerotinia sclerotiorum: Effect of carbon source on the isoenzymatic pattern. Riv. Patol. Veg. 2:111-123.

Ferrari, S., Sella, L., Janni, M., De Lorenzo, G., Favaron, F., and D'Ovidio, R. 2012. Transgenic expression of polygalacturonase-inhibiting proteins in Arabidopsis and wheat increases resistance to the flower pathogen Fusarium graminearum. Plant Biol Stuttg 14 (Suppl 1):31-38.

Fierens, E., Gebruers, K., Courtin, C. M., and Delcour, J. A. 2008. Xylanase inhibitors bind to nonstarch polysaccharides. J. Agric. Food Chem. 56: 564-570.

Giovane, A., Servillo, L., Balestrieri, C., Raiola, A., D’Avino, R., Tamburrini, M., Ciardiello, M. A., and Camardella, L. 2004. Pectin methylesterase inhibitor. Biochim. Biophys. Acta 1696:245-252.

Gomez, L. D., Bristow, J. K., Statham, E. R., and McQueen-Mason, S. J. 2008. Analysis of saccharification in Brachypodium distachyon stems under mild conditions of hydrolysis. Biotechnol. Biofuels 1:15.

Horevaj, P., Milus, E. A., and Bluhm, B. H. 2011. A real-time qPCR assay to quantify Fusarium graminearum biomass in wheat kernels. J. Appl. Microbiol. 111:396-406.

Hou, C., Lv, T., Zhan, Y., Peng, Y., Huang, Y., Jiang, D., and Weng, X. 2015. Overexpression of the RIXI xylanase inhibitor improves disease resistance to the fungal pathogen, Magnaporthe oryzae, in rice. Plant Cell Tiss. Org. 120:167-177.

Isshiki, A., Akimitsu, K., Yamamoto, M., and Yamamoto, H. 2001. Endopolygalacturonase is essential for citrus black rot caused by Alternaria citri but not brown spot caused by Alternaria alternata. Mol. Plant-Microbe Interact. 14:749-757.

Janni, M., Sella, L., Favaron, F., Blechl, A. E., De Lorenzo, G., and D'Ovidio, R. 2008. The expression of a bean PGIP in transgenic wheat confers increased resistance to the fungal pathogen Bipolaris sorokiniana. Mol. Plant-Microbe Interact. 21:171-177.
Juge, N. 2006. Plant protein inhibitors of cell wall degrading enzymes. Trends Plant Sci. 11:359-367.

Kalunke, R. M., Janni, M., Benedettelli, S., and D’Ovidio, R. 2013. Using biolistics and hybridization to combine multiple glycosidase inhibitor transgenes in wheat. Euphytica 194:443-457.

Kalunke, R. M., Tundo, S., Benedetti, M., Cervone, F., De Lorenzo, G., and D'Ovidio, R. 2015. An update on polygalacturonase-inhibiting protein (PGIP), a leucine-rich repeat protein that protects crop plants against pathogens. Front. Plant Sci. 6:146.

Kubicek, C. P., Starr, T. L., and Glass, N. L. 2014. Plant cell wall-degrading enzymes and their secretion in plant-pathogenic fungi. Annu. Rev. Phytopathol. 52:427-451.

Leckie, F., Mattei, B., Capodicasa, C., Hemmings, A., Nuss, L., Aracri, B., De Lorenzo, G., and Cervone, F. 1999. The specificity of polygalacturonaseinhibiting protein (PGIP): A single amino acid substitution in the solventexposed $\beta$-strand/ $\beta$-turn region of the leucine-rich repeats (LRRs) confers a new recognition capability. EMBO J. 18:2352-2363.

Li, R., Rimmer, R., Buchwaldt, L., Sharpe, A. G., Séguin-Swartz, G., and Hegedus, D. D. 2004. Interaction of Sclerotinia sclerotiorum with Brassica napus: Cloning and characterization of endo- and exo-polygalacturonases expressed during saprophytic and parasitic modes. Fungal Genet. Biol. 41 754-765.

Limberg, G., Körner, R., Buchholt, H. C., Christensen, T. M., Roepstorff, P., and Mikkelsen, J. D. 2000. Analysis of different de-esterification mechanisms for pectin by enzymatic fingerprinting using endopectin lyase and endopolygalacturonase II from A. niger. Carbohydr. Res. 327: 293-307.

Lionetti, V., Cervone, F., and Bellincampi, D. 2012. Methyl esterification of pectin plays a role during plant-pathogen interactions and affects plant resistance to diseases. J. Plant Physiol. 169:1623-1630.

Lionetti, V., Francocci, F., Ferrari, S., Volpi, C., Bellincampi, D., Galletti, R., D’Ovidio, R., De Lorenzo, G., and Cervone, F. 2010. Engineering the cell wall by reducing de-methyl-esterified homogalacturonan improves saccharification of plant tissues for bioconversion. Proc. Natl. Acad. Sci. U.S.A. 107:616-621.

Lionetti, V., Giancaspro, A., Fabri, E., Giove, S. L., Reem, N., Zabotina, O. A., Blanco, A., Gadaleta, A., and Bellincampi, D. 2015. Cell wall traits as potential resources to improve resistance of durum wheat against Fusarium graminearum. BMC Plant Biol. 15:6.

Lionetti, V., Raiola, A., Camardella, L., Giovane, A., Obel, N., Pauly, M., Favaron, F., Cervone, F., and Bellincampi, D. 2007. Overexpression of pectin methylesterase inhibitors in Arabidopsis restricts fungal infection by Botrytis cinerea. Plant Physiol. 143:1871-1880.

Lionetti, V., Raiola, A., Cervone, F., and Bellincampi, D. 2014. Transgenic expression of pectin methylesterase inhibitors limits tobamovirus spread in tobacco and Arabidopsis. Mol. Plant Pathol. 15:265-274.

Moscetti, I., Faoro, F., Moro, S., Sabbadin, D., Sella, L., Favaron, F., and D'Ovidio, R. 2015. The xylanase inhibitor TAXI-III counteracts the necrotic activity of a Fusarium graminearum xylanase in vitro and in durum wheat transgenic plants. Mol. Plant Pathol. 16:583-592.

Moscetti, I., Tundo, S., Janni, M., Sella, L., Gazzetti, K., Tauzin, A., Giardina, T., Masci, S., Favaron, F., and D'Ovidio, R. 2013. Constitutive expression of the xylanase inhibitor TAXI-III delays Fusarium head blight symptoms in durum wheat transgenic plants. Mol. Plant-Microbe Interact. 26:1464-1472.

Oeser, B., Heidrich, P. M., Müller, U., Tudzynski, P., and Tenberge, K. B. 2002. Polygalacturonase is a pathogenicity factor in the Claviceps purpurea/rye interaction. Fungal Genet. Biol. 36:176-186.

Pařenicová, L., Benen, J. A., Kester, H. C. M., and Visser, J. 2000. pgaA and pgaB encode two constitutively expressed endopolygalacturonases of Aspergillus niger. Biochem. J. 345:637-644.

Pelloux, J., Rustérucci, C., and Mellerowicz, E. J. 2007. New insights into pectin methylesterase structure and function. Trends Plant Sci. 12:267277

Raiola, A., Sella, L., Castiglioni, C., Balmas, V., and Favaron, F. 2008. A single amino acid substitution in highly similar endo-PGs from Fusarium verticillioides and related Fusarium species affects PGIP inhibition. Fungal Genet. Biol. 45:776-789.

Rodriguez-Palenzuela, P., Burr, T. J., and Collmer, A. 1991. Polygalacturonase is a virulence factor in Agrobacterium tumefaciens biovar 3 . J. Bacteriol. 173:6547-6552.

Roper, M. C., Greve, L. C., Warren, J. G., Labavitch, J. M., and Kirkpatrick, B. C. 2007. Xylella fastidiosa requires polygalacturonase for colonization and pathogenicity in Vitis vinifera grapevines. Mol. Plant-Microbe Interact. 20:411-419.

Salvi, G., Giarrizzo, F., De Lorenzo, G., and Cervone, F. 1990. A polygalacturonase-inhibiting protein in the flowers of Phaseolus vulgaris L. J. Plant Physiol. 136:513-518. 
Sambrook, J., Fritsch, E. F., and Maniatis, T. 1989. Molecular Cloning: A Laboratory Manual, 2nd Ed. Cold Spring Harbor Laboratory Press, Cold Spring Harbor, NY, U.S.A.

Scott, T. A., Jr., and Melvin, E. H. 1953. Determination of dextran with anthrone. Anal. Chem. 25:1656-1661.

Sella, L., Castiglioni, C., Paccanaro, M. C., Janni, M., Schäfer, W., D'Ovidio, R., and Favaron, F. 2016. Involvement of fungal pectin methylesterase activity in the interaction between Fusarium graminearum and wheat. Mol. Plant-Microbe Interact. 29:258-267.

Sella, L., Castiglioni, C., Roberti, S., D’Ovidio, R., and Favaron, F. 2004. An endo-polygalacturonase (PG) of Fusarium moniliforme escaping inhibition by plant polygalacturonase-inhibiting proteins (PGIPs) provides new insights into the PG-PGIP interaction. FEMS (Fed. Eur. Microbiol. Soc.) Microbiol. Lett. 240:117-124.

Shieh, M. T., Brown, R. L., Whitehead, M. P., Cary, J. W., Cotty, P. J., Cleveland, T. E., and Dean, R. A. 1997. Molecular genetic evidence for the involvement of a specific polygalacturonase, $\mathrm{P} 2 \mathrm{c}$, in the invasion and spread of Aspergillus flavus in cotton bolls. Appl. Environ. Microbiol. 63:3548-3552.

Siah, A., Deweer, C., Duyme, F., Sanssené, J., Durand, R., Halama, P., and Reignault, P. 2010. Correlation of in planta endo-beta-1,4-xylanase activity with the necrotrophic phase of the hemibiotrophic fungus Mycosphaerella graminicola. Plant Pathol. 59:661-670.

Spadoni, S., Zabotina, O., Di Matteo, A., Mikkelsen, J. D., Cervone, F., De Lorenzo, G., Mattei, B., and Bellincampi, D. 2006. Polygalacturonaseinhibiting protein interacts with pectin through a binding site formed by four clustered residues of arginine and lysine. Plant Physiol. 141: 557-564.

Tai, T. H., and Tanksley, S. D. 1990. A rapid and inexpensive method for isolation of total DNA from dehydrated plant tissue. Plant Mol. Biol. Rep. 8:297-303.

Tans-Kersten, J., Huang, H., and Allen, C. 2001. Ralstonia solanacearum needs motility for invasive virulence on tomato. J. Bacteriol. 183: 3597-3605. ten Have, A., Mulder, W., Visser, J., and van Kan, J. A. 1998. The endopolygalacturonase gene Bcpgl is required for full virulence of Botrytis cinerea. Mol. Plant-Microbe Interact. 11:1009-1016.

Tundo, S., Moscetti, I., Faoro, F., Lafond, M., Giardina, T., Favaron, F., Sella, L., and D'Ovidio, R. 2015. Fusarium graminearum produces different xylanases causing host cell death that is prevented by the xylanase inhibitors XIP-I and TAXI-III in wheat. Plant Sci. 240:161-169.

Updegraff, D. M. 1969. Semimicro determination of cellulose in biological material. Anal. Biochem. 32:420-424.

Vogel, J. 2008. Unique aspects of the grass cell wall. Curr. Opin. Plant Biol. 11:301-307.

Volpi, C., Janni, M., Lionetti, V., Bellincampi, D., Favaron, F., and D'Ovidio, R. 2011. The ectopic expression of a pectin methyl esterase inhibitor increases pectin methyl esterification and limits fungal diseases in wheat. Mol. Plant-Microbe Interact. 24:1012-1019.

Volpi, C., Raiola, A., Janni, M., Gordon, A., O'Sullivan, D. M., Favaron, F., and D'Ovidio, R. 2013. Claviceps purpurea expressing polygalacturonases escaping PGIP inhibition fully infects PvPGIP2 wheat transgenic plants but its infection is delayed in wheat transgenic plants with increased level of pectin methyl esterification. Plant Physiol. Biochem. 73:294-301.

Wang, A., Wei, X., Rong, W., Dang, L., Du, L. P., Qi, L., Xu, H. J., Shao, Y. and Zhang, Z. 2015a. GmPGIP3 enhanced resistance to both take-all and common root rot diseases in transgenic wheat. Funct. Integr. Genomics $15: 375-381$.

Wang, R., Lu, L., Pan, X., Hu, Z., Ling, F., Yan, Y., Liu, Y., and Lin, Y 2015 b. Functional analysis of OsPGIP1 in rice sheath blight resistance. Plant Mol. Biol. 87:181-191.

Willats, W. G., McCartney, L., Mackie, W., and Knox, J. P. 2001. Pectin: Cell biology and prospects for functional analysis. Plant Mol. Biol. 47: 9-27.

Xin, Z., Wang, Q., Yu, Z., Hu, L., Li, J., Xiang, C., Wang, B., and Lou, Y. 2014. Overexpression of a xylanase inhibitor gene, OsHI-XIP, enhances resistance in rice to herbivores. Plant Mol. Biol. Rep. 32:465-475. 